\title{
Matrix Metalloproteinase-Targeted Imaging of Lung Inflammation and Remodeling
}

\author{
Reza Golestani ${ }^{1,2}$, Mahmoud Razavian ${ }^{1,2}$, Yunpeng Ye ${ }^{1,2}$, Jiasheng Zhang ${ }^{1,2}$, Jae-Joon Jung ${ }^{1,2}$, Jakub Toczek ${ }^{1,2}$, \\ Kiran Gona ${ }^{1,2}$, Hye-Yeong Kim ${ }^{1,2}$, Jack A. Elias ${ }^{3}$, Chun Geun Lee ${ }^{3}$, Robert J. Homer ${ }^{2,4}$, and Mehran M. Sadeghi ${ }^{1,2}$ \\ ${ }^{1}$ Section of Cardiovascular Medicine and Cardiovascular Research Center, Yale School of Medicine, New Haven, Connecticut; \\ ${ }^{2}$ VA Connecticut Healthcare System, West Haven, Connecticut; ${ }^{3}$ Brown University, Providence, Rhode Island; and ${ }^{4}$ Department of \\ Pathology, Yale School of Medicine, New Haven, Connecticut
}

Imaging techniques for detection of molecular and cellular processes that precede or accompany lung diseases are needed. Matrix metalloproteinases (MMPs) play key roles in the development of pulmonary pathology. The objective of this study was to investigate the feasibility of in vivo MMP-targeted molecular imaging for detection of lung inflammation and remodeling. Methods: Lung-specific IL-13 transgenic (Club cell 10-kDa protein [CC10]-IL-13 Tg) mice and wild-type littermates were used in this study. Lung structure, gene expression, and MMP activity were assessed by histology, real-time reverse transcription polymerase chain reaction, Western blotting, and zymography. MMP activation was imaged by in vivo small-animal SPECT/CT followed by ex vivo planar imaging. Signal specificity was addressed using a control tracer. The correlation between in vivo MMP signal and gene expression was addressed. Results: CC10-IL-13 Tg mice developed considerable pulmonary tissue remodeling and inflammation. CD68, MMP-12, and MMP-13 were significantly higher in CC10-IL$13 \mathrm{Tg}$ lungs. On in vivo small-animal SPECT/CT and ex vivo planar images, the MMP signal was significantly higher in the lungs of CC10IL-13 Tg mice than wild-type animals. Furthermore, a nonbinding analog tracer showed significantly lower accumulation in CC10-IL-13 $\mathrm{Tg}$ lungs relative to the specific tracer. There was a significant correlation between small-animal SPECT/CT-derived MMP signal and CD68 expression in the lungs $(r=0.70, P<0.01)$. Conclusion: Small-animal SPECT/CT-based MMP-targeted imaging of the lungs is feasible and reflects pulmonary inflammation. If validated in humans, molecular imaging of inflammation and remodeling can potentially help early diagnosis and monitoring of the effects of therapeutic interventions in pulmonary diseases.

Key Words: molecular imaging; chronic obstructive pulmonary disease; interleukin-13; SPECT; matrix metalloproteinases

J Nucl Med 2017; 58:138-143

DOI: 10.2967/jnumed.116.176198

$\mathbf{P}$ ulmonary diseases are major causes of morbidity and mortality in the United States. Chronic obstructive pulmonary disease (COPD) alone affects approximately 16 million people and is the fourth leading cause of death in this country (1-3). Inflammation

Received Mar. 24, 2016; revision accepted Jul. 5, 2016

For correspondence or reprints contact: Mehran M. Sadeghi, Yale Cardiovascular Research Center, 300 George Street, \#770F, New Haven, CT 06511.

E-mail: mehran.sadeghi@yale.edu

Published online Jul. 28, 2016.

COPYRIGHT (C 2017 by the Society of Nuclear Medicine and Molecular Imaging. is a common and key early player in the pathogenesis of pulmonary diseases, and depending on the trigger and context can lead to COPD, asthma, pulmonary fibrosis, or pulmonary hypertension. Each of these pulmonary diseases is characterized by the presence of an inflammatory infiltrate and a combination of alveolar, airway, and vascular remodeling; airways hyperresponsiveness; mucus cell metaplasia; and fibrosis. The interplay of cytokines and growth factors with pulmonary cells mediates the development of pulmonary inflammation and remodeling. An interleukin-13 (IL-13)/transforming growth factor- $\beta$ axis appears to be especially important in the pathogenesis of pulmonary fibrosis, asthma, and COPD and is under investigation for treatment of remodeling in pulmonary diseases (4-7).

Currently, the assessment of progression and response to treatment in COPD and many other pulmonary diseases is based on clinical manifestations, spirometry, and anatomic imaging. This assessment overlooks the underlying processes that precede or accompany clinical and physiologic alterations. In vivo detection of molecular and cellular processes that are involved in the pathogenesis of pulmonary disease can lead to early diagnosis and help track the effect of therapeutic interventions. Matrix metalloproteinases (MMPs) play prominent roles in pulmonary inflammation and remodeling and have recently emerged as promising novel therapeutic targets in lung disease $(8-11)$. Several proinflammatory cytokines (e.g., IL-13) and growth factors induce MMP expression and activation (11-13). Several MMPs are upregulated in response to stimuli (such as smoking) that promote pulmonary inflammation, fibrosis, and remodeling (14). As such, detection and quantification of MMP activation in the lungs can potentially provide unique diagnostic and prognostic insight into the course of obstructive and restrictive lung diseases.

In this study, we sought to address the feasibility of MMPtargeted molecular imaging for detection of lung inflammation and remodeling. Using transgenic mice that exclusively express IL-13 in the lungs (IL-13 Tg) and a ${ }^{99 m}$ Tc-labeled macrocyclic MMP-targeted tracer in conjunction with small-animal SPECT/ CT imaging, we established the feasibility of MMP imaging in lung pathology and investigated the biologic correlates of the signal in vivo, demonstrating a strong correlation between MMP activation and lung inflammation.

\section{MATERIALS AND METHODS}

\section{Reagents}

Reagents were purchased from Sigma-Aldrich unless otherwise specified. Recombinant human MMPs were obtained from R\&D Systems. 


\section{Animal Mode}

Club cell 10-kDa protein (CC10)-IL-13 Tg mice $(n=21$; median age, $10 \mathrm{wk}$; age range, 8-12 wk) (15) and their transgene-negative, wild-type (WT) littermates $(n=8)$ were used in this study. In CC10IL-13 Tg mice, IL-13 is specifically targeted to the lung using the CC10 promoter. All experiments were performed under protocols approved by Yale University and VA Connecticut Institutional Animal Care and Use Committees.

\section{Tracers}

The precursor of RP805, a ${ }^{99 \mathrm{~m}} \mathrm{Tc}-$ labeled tracer with specificity for activated MMPs (16) (Supplemental Fig. 1A; supplemental materials are available at http://jnm.snmjournals.org), was synthetized in-house as described previously (16). Briefly, the RP805 precursor was synthesized from an antisuccinic acid analog, that is, (2R,3S)-3-(tertbutyoxycarbonyl)-2-iso-butylhe-5-enoic acid, in multiple steps. The final product was purified by reversed-phase high-performance liquid chromatography (RP-HPLC), identified by analytic RP-HPLC and liquid chromatography-mass spectrometry (data not shown), and tested for its binding affinity toward a set of recombinant human MMPs (Supplemental Table 1). A new, nonbinder amide analog of RP805 precursor (Supplemental Fig. 1B) was synthesized by replacing the hydroxamate group of RP805 precursor with the $N$-methyl amide group. As expected, this compound did not show any significant inhibition against recombinant human MMP-12 due to lack of the hydroxamate group. Both compounds were successfully labeled using a non- $\mathrm{SnCl}_{2}$ formulation (17) to give the desired ${ }^{99 \mathrm{~m}} \mathrm{Tc}$-labeled products in high radiochemical yield and radiochemical purity (99\%) as confirmed by radio-HPLC.

\section{Affinity Measurement}

Affinity measurements were based on inhibition of MMP-mediated cleavage of a fluorogenic substrate, (7-methoxycoumarin-4-yl)acetylPro-Leu-Gly-Leu-Dpa-Ala-Arg- $\mathrm{NH}_{2}$, as described previously (18).

\section{Imaging}

Small-animal SPECT/CT imaging was performed as described previously with some modification (19). RP805 (37 MBq) or its ${ }^{99 \mathrm{~m}}$ Tc-labeled amide analog, as a negative control tracer, was administered through a jugular vein catheter. Images were acquired at $2 \mathrm{~h}$ after injection with a small-animal imaging system (X-SPECT; $\gamma$-MedicaIdeas) equipped with 1-mm pinhole collimators. Mice anesthetized with inhaled isoflurane $(1 \%)$ were placed in a fixed position on the animal bed, and small-animal SPECT imaging was performed using the following parameters: 64 projections, $30 \mathrm{~s}$ per projection; $360^{\circ}$ rotation; matrix, $82 \times 82$; and $140-\mathrm{keV}$ photopeak $\pm 10 \%$ window. Immediately after small-animal SPECT imaging, a noncontrast $\mathrm{CT}$ image was acquired to provide anatomic data for image quantification. After small-animal SPECT and CT image acquisition, the animals were euthanized, and lungs, heart, and aorta were harvested for planar imaging and histology (the right lung was used for ex vivo imaging, and the left lung was snapfrozen in optimal-cutting-temperature compound for further RNA and protein analysis). Small-animal SPECT data were reconstructed and analyzed as described previously (19). For image analysis, spheric regions of interest were drawn in the lungs on the basis of small-animal CT images. Total activity within a region of interest was calculated on the basis of known activities of reference point sources. The values were corrected for both injected dose and background (posterior to thoracic vertebrae). Uptake was presented as percentage injected dose per $\mathrm{cm}^{3}\left(\% \mathrm{ID} / \mathrm{cm}^{3}\right)$.

\section{Histology and Real-Time Reverse Transcription Polymerase Chain Reaction}

Hematoxylin and eosin staining was performed on 5- $\mu$ m-thick sections of formalin-fixed tissue (from animals perfused with saline) according to standard techniques. For real-time reverse transcription polymerase chain reaction (20), total RNA was isolated from equal tissue volumes of fresh-frozen lung tissue. The following Taqman probes were used: CD68 (Mm00839636_g1), MMP-2 (Mm00439506_m1), MMP-9 (Mm00442991_m1), MMP-12 (Mm00500554_m1), MMP13 (Mm00439491_m1), TIMP-1 (Mm01341361_m1), TIMP-2 (Mm00441825_m1), and TIMP-3 (Mm00441827_m1). For each gene, gene expression is shown relative to average gene expression in WT animals.

\section{Gel Zymography and Western Blotting}

Proteins were extracted from equal volumes of snap-frozen lung tissue $\left(0.2 \mathrm{~mm}^{3}\right)$ using a lysis buffer containing $0.5 \%$ Triton $\mathrm{X}-100$ and proteinase inhibitor cocktail (Roche Diagnostics). Gelatinase gel zymography was performed on these protein extracts using Novex $10 \%$ Zymogram (gelatin) Gels (Life Technologies) according to the manufacturer's instructions. Western blotting was performed using an anti-CD68 antibody (Serotec) and quantified as described previously (21). For Western blotting analysis, 1 sample used on 2 separate gels served as internal control. Band intensities were measured in relation to this internal control.

\section{Tissue Binding Specificity}

Lung tissue sections from CC10-IL-13 Tg mice were exposed to 1,000 -fold (on a molar basis) nonlabeled RP805 precursor or nothing in $100 \mu \mathrm{L}$ of phosphate-buffered saline at $37^{\circ} \mathrm{C}$ for $2 \mathrm{~min}$, followed by ${ }^{99 \mathrm{~m} T c}$-labeled RP805 $(185 \mathrm{kBq})$ for $15 \mathrm{~min}$ in triplicate. After 3 washes in phosphate-buffered saline, the residual activity was measured using a $\gamma$-well counter (PerkinElmer). The data were corrected for protein content and background and are presented relative to RP805 alone.

\section{Statistical Analysis}

Data are presented as mean \pm SD. Group comparisons were based on the 2-tailed Student $t$ test (for equal variances) or paired $t$ test (for paired samples). The Pearson correlation coefficient was used to test the correlation between variables. Statistical analysis was performed using GraphPad Prism 6.0 (GraphPad Software, Inc.).

\section{RESULTS}

\section{Lung Inflammation and Remodeling in CC10-IL-13 Tg Mice}

IL-13 expression in CC10-IL-13 Tg mice led to considerable enlargement of the lungs compared with age-matched WT animals. This difference was readily detectable in animals aged 8-12 wk (Fig. 1A). Histologic analysis of the lungs of CC10-IL-13 Tg mice demonstrated loss of normal lung architecture, with alveolar enlargement, inflammatory cell (including macrophage) infiltration, and airway remodeling including airway fibrosis and respiratory epithelial hypertrophy (Figs. 1B-1D). In line with enhanced tissue inflammation, CD68 expression was significantly increased in CC10-IL-13 Tg lungs (Fig. 2). This increase was associated with changes in MMP expression (Supplemental Fig. 2). As such, compared with WT animals, MMP-12 and MMP-13 were significantly higher in CC10-IL-13 Tg mice (Supplemental Figs. 2C and 2D). MMP-2 expression was not different between WT and transgenic animals whereas for MMP-9 messenger RNA there was a nonstatistically significant trend in the opposite direction. Pointing to the complexity of the regulation of MMP function in vivo, which depends on MMP expression level, activation state, and presence of tissue inhibitors of MMPs (TIMPs), TIMP-1 expression was significantly higher in CC10-IL-13 Tg lungs (Supplemental Fig. 2E) whereas there was no difference between transgenic and WT animals in TIMP-2 and TIMP-3 messenger RNA expression. Evaluation of lung extracts by gel zymography showed distinct bands corresponding to MMP-2 and MMP-9 gelatinolytic activity. In this 


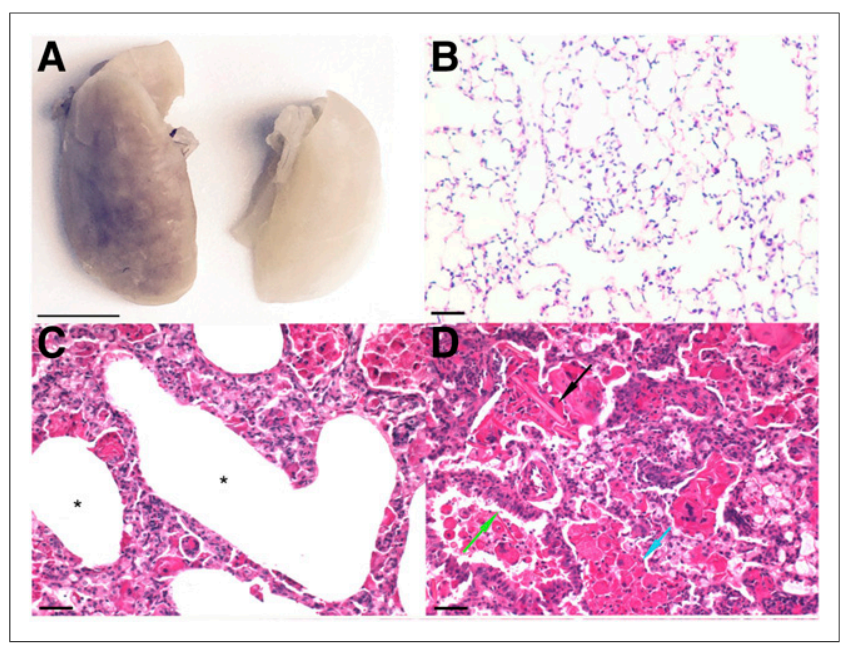

FIGURE 1. Tissue remodeling in CC10-IL-13 Tg lungs. (A) Representative photographs of CC10-IL-13 Tg (left) and transgene-negative, WT lungs (right). Scale bar $=10 \mathrm{~mm}$. (B) Representative hematoxylin and eosin staining of lung from WT mouse demonstrating normal tissue architecture. Scale bar $=100 \mu \mathrm{m}$. (C and D) Representative examples of hematoxylin and eosin staining of CC10-IL-13 Tg lungs, demonstrating alveolar enlargement and remodeling (asterisk), macrophage infiltration (blue arrow), crystal deposition within alveolar space and macrophages (black arrows), and airway remodeling and hyperplasia (green arrow). Scale bar $=100 \mu \mathrm{m}$.

regard, although there was no difference in MMP-9 activity between the 2 groups of animals, MMP-2 activity (both the pro-MMP and the active forms) was significantly higher in CC10-IL-13 Tg lungs than in those from WT animals (Figs. 3A-3C). Similarly, evaluation of tissue lysates by casein gel zymography showed significantly higher MMP-12 enzymatic activity in CC10-IL-13 Tg mice (Fig. 3D).

\section{MMP Imaging of Lungs}

To address the feasibility of molecular imaging of MMP activation in the lungs, CC10-IL-13 Tg and WT mice underwent in vivo microSPECT/CT imaging with RP805, a ${ }^{99 m}$ Tc-labeled tracer with specificity for activated MMPs. On visual analysis of fused images, the MMP signal was readily detectable in CC10-IL-13 Tg lungs but not in WT animals (Fig. 4). This was confirmed by quantitative analysis
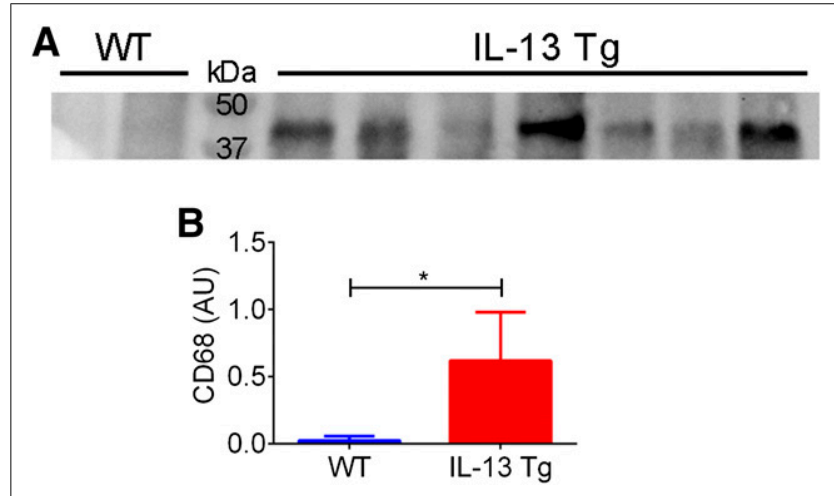

FIGURE 2. Tissue inflammation in CC10-IL-13 Tg lungs. Examples of Western blot images (A) and quantification of CD68 expression (B) in WT $(n=5)$ and CC10-IL-13 $\operatorname{Tg}(n=12)$ lungs. Data are from equal volumes of lung tissue and are reported as mean \pm SD. ${ }^{*} P<0.01$.

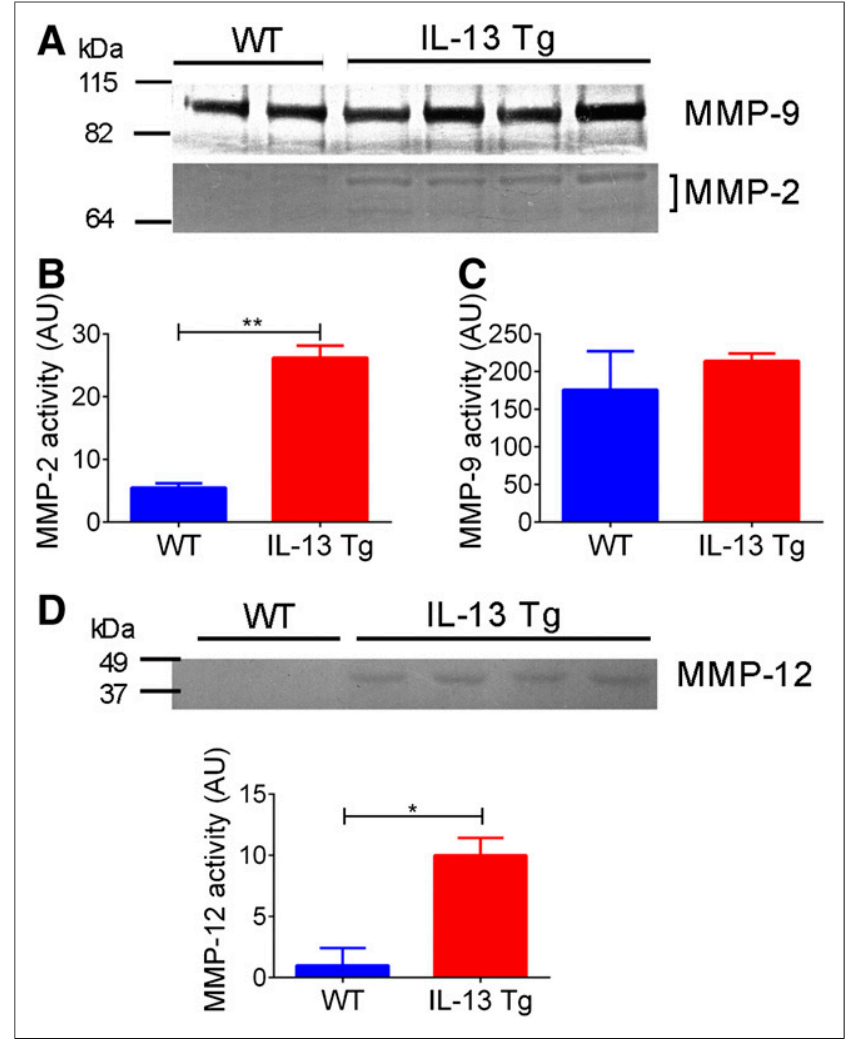

FIGURE 3. MMP activity. (A) Gelatin gel zymography of lung extracts from WT and CC10-IL-13 Tg mice, demonstrating proteolytic bands at $95 \mathrm{kDa}$ and 72 and $66 \mathrm{kDa}$, corresponding to MMP-9 and MMP-2, respectively. (B and $C$ ) Intensity quantification of MMP-2- (B) and MMP9-related (C) bands. (D) Casein gel zymography, demonstrating 45-kDa MMP-12 proteolytic activity (top) and quantification of MMP-12 band intensity (bottom) of lung extracts from WT and CC10-IL-13 Tg transgenic mice. ${ }^{\star} P<0.05 .{ }^{\star \star} P<0.01$. AU $=$ arbitrary units.

of RP805 uptake in the lungs, which showed significantly higher uptake of the tracer in CC10-IL-13 Tg mice than WT animals $\left(6.90 \pm 1.76, n=13\right.$ vs. $3.06 \pm 1.33 \% \mathrm{ID} / \mathrm{cm}^{3}, n=6$, respectively, for CC10-IL-13 Tg and WT mice; $P<0.001$ ).

RP805 uptake in CC10-IL-13 Tg lungs was confirmed by ex vivo planar imaging. Similar to in vivo images, planar images of harvested lungs showed significantly higher MMP signal in CC10IL-13 Tg mice $\left(0.17 \pm 0.04\right.$ vs. $0.09 \pm 0.02 \mathrm{cpp} / \mathrm{mm}^{2} / \mathrm{MBq} ; P<$ 0.001) (Supplemental Figs. 3A-3D). There was a significant correlation between small-animal SPECT-derived and planar imagederived tracer uptake $(r=0.79, P<0.001)$, validating our quantification methodology (Supplemental Fig. 3E).

\section{Specificity}

The enhanced endothelial permeability associated with inflammation can lead to nonspecific uptake of the tracer. To address the specificity of RP805 uptake in our model, we developed a new nonbinding analog by changing the hydroxamate group with $N$-methyl amide group (amide analog, Supplemental Fig. 1B). Small-animal SPECT/CT imaging of CC10-IL-13 Tg mice using the amide analog showed significantly lower accumulation of this control tracer in the lungs $\left(2.87 \% \mathrm{ID} / \mathrm{cm}^{3} \pm 1.61 ; P<0.001\right)$ than RP805 (Fig. 4). Similarly, ex vivo planar imaging showed significantly lower uptake of this amide analog tracer in CC10-IL-13 Tg 


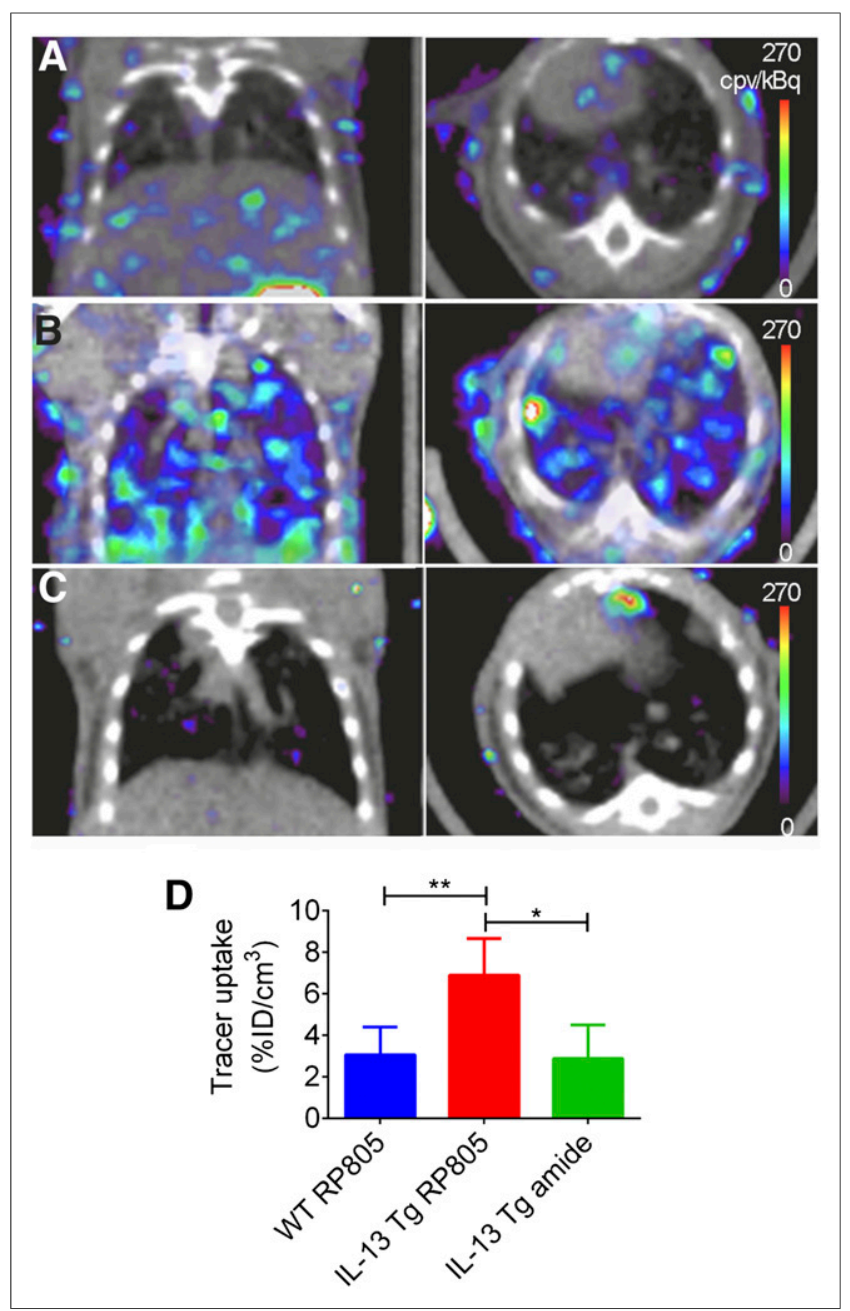

FIGURE 4. In vivo small-animal SPECT/CT imaging of MMP activation. (A-C) Examples of coronal (left) and transversal (right) views of fused small-animal SPECT/CT images of WT mice injected with RP805 (A) and CC10-IL-13 Tg mice injected with RP805 (B) or its control, amide analog tracer (C). (D) Small-animal SPECT-derived quantification tracer uptake in lungs. $n=6,13$, and 5 , respectively, for WT mice injected with RP805 and $\mathrm{CC} 10-\mathrm{IL}-13 \mathrm{Tg}$ mice injected with RP805 or amide, control analog. ${ }^{\star} P<0.01$. ${ }^{\star \star} P<0.001 . \mathrm{cpv}=$ counts per voxel; ID $=$ injected dose.

lungs $\left(0.09 \mathrm{cpp} / \mathrm{mm}^{2} / \mathrm{MBq} \pm 0.03 ; P<0.01\right)$ than $\mathrm{RP} 805$ (Supplemental Fig. 3). Preincubation with nonlabeled precursor significantly blocked RP805 binding to CC10-IL-13 Tg lung sections, further confirming RP805 binding specificity (Supplemental Fig. 4).

\section{Correlates of MMP Signal in Lungs}

MMPs are produced by several cells in the lungs. The extracellular nature of many MMPs (22), activated MMP uptake by adjacent cells (23), and the absence of highly selective antibodies hamper our ability to unequivocally link the MMP signal to specific cells in the lungs. Given the role of inflammatory cells as major sources of MMP production and activation in the lungs, we investigated a potential correlation between MMP activation detected by molecular imaging and macrophage content of the tissue. Not surprisingly, we found a significant correlation between RP805 signal in vivo on small-animal SPECT/CT images and CD68 expression quantified by Western blotting ( $r=0.70, P<0.01$, Fig. 5 ). Similarly, there was a significant correlation between MMP-12 gene expression and RP805 uptake ( $r=0.71, P<0.01$, Supplemental Fig. 5).

\section{DISCUSSION}

This study established the feasibility of in vivo MMP-targeted imaging for the detection of protease activity within the lungs, demonstrating a correlation between small-animal SPECT/CTderived MMP signal and lung inflammation. Significantly lower uptake of a nonavid, control tracer in the lungs of CC10-IL-13 $\mathrm{Tg}$ mice, which exhibit considerable tissue remodeling and inflammatory cell infiltration and enhanced MMP activation, ruled out the possibility that RP805 signal in transgenic mice is related to enhanced permeability or nonspecific binding to lung tissue. Transgenic expression of IL-13 in the lungs, whether constitutive or inducible, leads to considerable pulmonary inflammation, airway and alveolar remodeling, fibrosis, and respiratory failure $(15,24,25)$. CC10-IL-13 Tg mice show a spectrum of phenotypes that mirror emphysema, idiopathic pulmonary fibrosis, and asthma in humans $(15,24)$. In line with the protease-antiprotease imbalance paradigm as the mediator of pulmonary remodeling in humans (24), these phenotypes in transgenic mice are mediated, at least in part, through stimulation of matrix remodeling via upregulation and activation of different members of MMP family. As such, the CC10IL-13 Tg mouse is an effective experimental model for molecular imaging studies of lung inflammation and remodeling.

Beyond symptoms, current evaluation of pulmonary pathology is predominantly based on physiologic measurements (e.g., pulmonary function testing) and anatomic imaging by chest x-ray and CT. The limitations of this approach are well recognized, because the results of these tests often lag behind cellular and molecular processes that underlie the development of the disease or resolution of pulmonary pathology in response to therapeutic interventions. Quantitative imaging techniques to detect pulmonary pathophysiology, to track effectiveness of therapeutic interventions, and to advance pulmonary research are much in need. Emerging CT- and MRI-based imaging techniques seek to measure gas trapping, lung volume, lung density, and airway and vessel morphology (26-29). However, these techniques are incapable of tracking key molecular and cellular

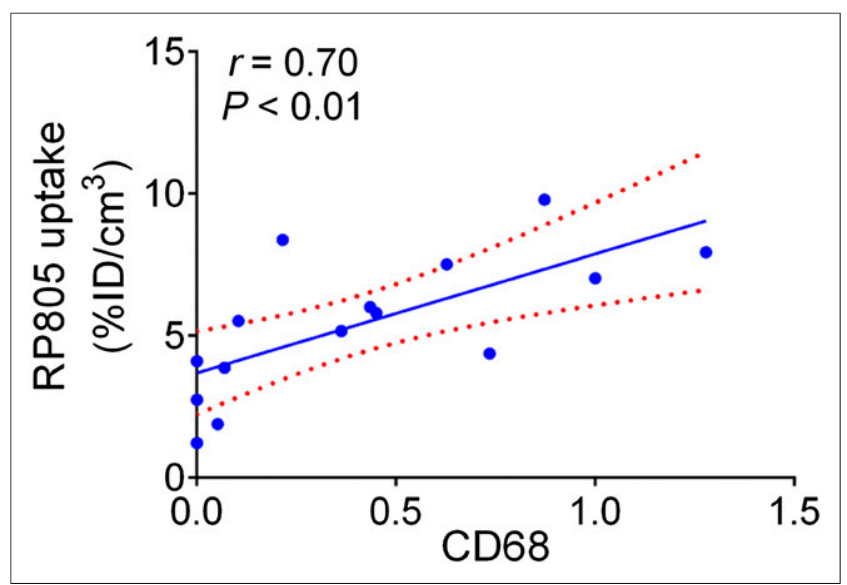

FIGURE 5. Correlation between small-animal SPECT-derived quantification of RP805 uptake and CD68 protein in arbitrary units in lungs from WT and CC10-IL-13 Tg mice imaged with RP805. Dotted lines represent $95 \%$ confidence interval. ID $=$ injected dose. 
processes. In this regard, molecular imaging is a valuable addition to the current arsenal of lung imaging techniques. The high sensitivity of radionuclide-based molecular imaging technology (i.e., SPECT and PET) allows the detection of targets in the nanomolar concentration range and is quite advantageous for this purpose.

Molecular imaging is routinely used in the clinic for detection, characterization, and staging of lung cancer. A few studies have explored other applications of molecular imaging in pulmonary pathology. As such, the short-term reproducibility of ${ }^{18}$ F-FDG PET in idiopathic pulmonary fibrosis has been demonstrated, but the biologic basis of the signal and its clinical usefulness remain to be determined (30). Similarly, it is suggested that PET imaging of somatostatin receptor with ${ }^{68} \mathrm{Ga}$-DOTANOC may be useful for the detection of fibroblast activity in idiopathic pulmonary fibrosis, because a correlation exists between ${ }^{68} \mathrm{Ga}$-DOTANOC signal and disease extent defined by high-resolution CT (31). In the preclinical setting, small-animal SPECT/CT imaging with ${ }^{111}$ In-DTPAA20FMDV2, an $\alpha_{v} \beta_{6}$ integrin-avid tracer, has shown promise for the detection of epithelial cell integrin expression (and possibly collagen content) in a murine model of bleomycin-induced pulmonary fibrosis (32). Yet, despite the prevalence and importance of inflammation in pulmonary pathology, techniques for specific imaging of lung inflammation are lacking. The central role of MMPs in pulmonary inflammation and remodeling suggests that MMP-targeted imaging maybe particularly powerful in this regard.

MMPs are a family of zinc-dependent proteases that regulate matrix remodeling and inflammation through degradation of extracellular matrix proteins, modulation of cell signaling, and regulation of chemokines and growth factor function $(22,33)$. Typically, MMPs are produced as inactive proenzymes, which undergo proteolytic activation to expose a catalytic site. MMP function is regulated at several levels, including gene expression, proenzyme activation, expression of inhibitors (e.g., TIMPs, $\alpha 2$-macroglobulin), macromolecular complex formation, and compartmentalization. This complexity of MMP biology is reflected in differences in gene expression between transgenic and WT animals and the discordance between MMP messenger RNA expression and activity for specific members of the MMP family. The lack of highly specific antibodies for the activated forms of MMPs and their complex biology, including the mismatch between the sites of protein synthesis and proteolytic activation, extracellular secretion, and uptake by neighboring cells (23), preclude accurate colocalization of activated MMPs with specific lung cells. Nevertheless, macrophages are the major source of MMP production in the lungs, although other intrinsic lung cells (e.g., epithelial cells) also contribute to this process (34). As such, the correlation between MMP signal detected in vivo and CD68 expression in our model is not surprising and is indeed consistent with similar observations in vascular and valvular pathology $(19,20,35)$. Although the relative value of imaging specific members of the MMP family versus MMP activation in general as an investigational tool can be debated, clinically this may be less relevant. A few tracers for ex vivo fluorescence-based optical imaging of specific members of the MMP family have been developed $(36,37)$. However, it is not clear whether the expression level of any specific member of the MMP family reaches the threshold for in vivo imaging, even using the most sensitive nuclear techniques. Whether imaging a specific member of the MMP family in vivo is feasible and more effective than targeting MMP activation in general remains to be empirically determined.
A potential pitfall in cardiothoracic molecular imaging is the effect of respiratory and cardiac motion on signal quantification. The confounding effects of this motion, which leads to an apparent reduction in the lung signal, is especially important for focal processes and smaller regions and lesions. As such, it is critical to ascertain the validity of signal quantification methodology for in vivo images. Addressing this issue, we showed a strong correlation between in vivo small-animal SPECT- and ex vivo planar imagingderived measures of RP805 uptake. Because of the diffuse nature of the pathology, respiratory motion correction of images appears to be less critical in IL-13 transgenic mice. However, this factor should be considered in molecular imaging of human lesions for which regional differences in disease activity may exist. Another potential confounding factor is the difference in lung structure, density, size, content, and cellularity between normal and pathologic conditions. Indeed, traditional housekeeping gene-based normalization methodologies are not as effective in comparing CC10-IL-13 Tg and WT animals. Therefore, we relied on volume-based measurements to compare and correlate imaging and biologic data in our animals. This approach rules out the possibility that the observed differences between transgenic and control animals are secondary to differences in lung volume and can be readily translated to clinical imaging. On the basis of the premise that changes in MMP activation precede structural changes in the lungs, MMP-targeted imaging could be used for tracking the effect of therapeutic interventions and possibly risk stratification in inflammatory pulmonary diseases. Similar to ${ }^{18}$ F-FDG PET studies, in such cases a threshold value (to be determined clinically) would distinguish the baseline expression in the normal tissue from upregulated signal in diseased lungs.

\section{CONCLUSION}

We have shown that in vivo MMP-targeted imaging of the lungs is feasible, and the signal reflects pulmonary inflammation. Because of the costs associated with new tracer development, a practical prerequisite for introduction of emerging molecular imaging techniques into the clinic is their applicability in a wide range of highly prevalent diseases. Unlike targets that are lung-specific, MMPs play a key role in cancer, inflammatory disorders, cardiovascular pathology, and a variety of lung diseases. Our data show a significant correlation between MMP activation and inflammation in the lungs. If validated in humans, MMP-targeted molecular imaging of inflammation and remodeling can potentially help early diagnosis and monitoring of the effects of therapeutic interventions in COPD, pulmonary fibrosis, and asthma. It will also be a valuable tool for discovery and development of antiinflammatory and antiproteolytic drugs in the preclinical and clinical settings.

\section{DISCLOSURE}

This work was supported by grants from the NIH (R01HL112992, R01-HL114703), Connecticut Department of Public Health (2016-0087), and Department of Veterans Affairs (I0BX001750). No other potential conflict of interest relevant to this article was reported.

\section{ACKNOWLEDGMENT}

We thank Dr. Terence Wu (Yale West Campus Analytical Core Laboratory) for his invaluable technical assistance. 


\section{REFERENCES}

1. Hilleman DE, Dewan N, Malesker M, Friedman M. Pharmacoeconomic evaluation of COPD. Chest. 2000;118:1278-1285.

2. Faulkner MA, Hilleman DE. The economic impact of chronic obstructive pulmonary disease. Expert Opin Pharmacother. 2002;3:219-228.

3. Raghu G. Interstitial lung disease: a clinical overview and general approach. In: Fishman AP, Elias JA, Fishman JA, et al., eds. Fishman's Pulmonary Diseases and Disorders. Vol. 2. New York, NY: Mc-Graw Hill Inc.; 1998:1037-1053.

4. Lee CG, Kang HR, Homer RJ, Chupp G, Elias JA. Transgenic modeling of transforming growth factor-beta(1): role of apoptosis in fibrosis and alveolar remodeling. Proc Am Thorac Soc. 2006;3:418-423.

5. Wills-Karp M. Interleukin-13 in asthma pathogenesis. Immunol Rev. 2004;202:175-190.

6. Halwani R, Al-Muhsen S, Al-Jahdali H, Hamid Q. Role of transforming growth factor-beta in airway remodeling in asthma. Am J Respir Cell Mol Biol. 2011;44:127133.

7. Corren J, Lemanske RF, Hanania NA, et al. Lebrikizumab treatment in adults with asthma. N Engl J Med. 2011;365:1088-1098.

8. Wu Y, Li J, Wu J, et al. Discovery of potent and selective matrix metalloprotease 12 inhibitors for the potential treatment of chronic obstructive pulmonary disease (COPD). Bioorg Med Chem Lett. 2012;22:138-143.

9. Magnussen H, Watz H, Kirsten A, et al. Safety and tolerability of an oral MMP-9 and -12 inhibitor, AZD1236, in patients with moderate-to-severe COPD: a randomised controlled 6-week trial. Pulm Pharmacol Ther. 2011;24:563-570.

10. $\mathrm{Li} \mathrm{W}, \mathrm{Li}$ J, Wu Y, et al. A selective matrix metalloprotease 12 inhibitor for potential treatment of chronic obstructive pulmonary disease (COPD): discovery of (S)-2-(8(methoxycarbonylamino)dibenzo[b,d]furan-3-sulfonamido)-3-methylbu tanoic acid (MMP408). J Med Chem. 2009;52:1799-1802.

11. Lagente V, Le Quement C, Boichot E. Macrophage metalloelastase (MMP-12) as a target for inflammatory respiratory diseases. Expert Opin Ther Targets. 2009; 13:287-295.

12. Xie S, Issa R, Sukkar MB, et al. Induction and regulation of matrix metalloproteinase12 in human airway smooth muscle cells. Respir Res. 2005;6:148.

13. Lanone S, Zheng T, Zhu Z, et al. Overlapping and enzyme-specific contributions of matrix metalloproteinases-9 and -12 in IL-13-induced inflammation and remodeling. J Clin Invest. 2002;110:463-474.

14. Churg A, Zhou S, Wright JL. Series "matrix metalloproteinases in lung health and disease": matrix metalloproteinases in COPD. Eur Respir J. 2012;39:197-209.

15. Zhu Z, Homer RJ, Wang Z, et al. Pulmonary expression of interleukin-13 causes inflammation, mucus hypersecretion, subepithelial fibrosis, physiologic abnormalities, and eotaxin production. J Clin Invest. 1999;103:779-788.

16. Su H, Spinale FG, Dobrucki LW, et al. Noninvasive targeted imaging of matrix metalloproteinase activation in a murine model of postinfarction remodeling. Circulation. 2005;112:3157-3167.

17. Liu S, Edwards DS, Harris AR, et al. Towards developing a non-SnCl2 formulation for RP444, a new radiopharmaceutical for thrombus imaging. J Pharm Sci. 2001;90:114-123.

18. Devel L, Rogakos V, David A, et al. Development of selective inhibitors and substrate of matrix metalloproteinase-12. J Biol Chem. 2006;281:11152-11160.

19. Jung JJ, Razavian M, Challa AA, et al. Multimodality and molecular imaging of matrix metalloproteinase activation in calcific aortic valve disease. $\mathrm{J} \mathrm{Nucl} \mathrm{Med}$. 2015;56:933-938.
20. Golestani R, Razavian M, Nie L, et al. Imaging vessel wall biology to predict outcome in abdominal aortic aneurysm. Circ Cardiovasc Imaging. 2014;8: $\mathrm{e} 002471$.

21. Nie L, Guo X, Esmailzadeh L, et al. Transmembrane protein ESDN promotes endothelial VEGF signaling and regulates angiogenesis. $J$ Clin Invest. 2013;123:5082-5097.

22. Iyer RP, Patterson NL, Fields GB, Lindsey ML. The history of matrix metalloproteinases: milestones, myths, and misperceptions. Am J Physiol Heart Circ Physiol. 2012;303:H919-H930.

23. Marchant DJ, Bellac CL, Moraes TJ, et al. A new transcriptional role for matrix metalloproteinase-12 in antiviral immunity. Nat Med. 2014;20:493-502.

24. Zheng T, Zhu Z, Wang Z, et al. Inducible targeting of IL-13 to the adult lung causes matrix metalloproteinase- and cathepsin-dependent emphysema. J Clin Invest. 2000;106:1081-1093.

25. Lee CG, Homer RJ, Zhu Z, et al. Interleukin-13 induces tissue fibrosis by selectively stimulating and activating transforming growth factor beta(1). $J$ Exp Med. 2001;194:809-821.

26. Coxson HO, Leipsic J, Parraga G, Sin DD. Using pulmonary imaging to move chronic obstructive pulmonary disease beyond FEV1. Am J Respir Crit Care Med. 2014;190:135-144.

27. Hersh CP, Washko G, Estepar RSJ, et al. Paired inspiratory-expiratory chest CT scans to assess for small airways disease in COPD. Respir Res. 2013;14:42.

28. Kirby M, Svenningsen S, Kanhere N, et al. Pulmonary ventilation visualized using hyperpolarized helium-3 and xenon-129 magnetic resonance imaging: differences in COPD and relationship to emphysema. J Appl Physiol. 2013;114: 707-715.

29. Rogers RM, Coxson HO, Sciurba FC, Keenan RJ, Whittall KP, Hogg JC. Preoperative severity of emphysema predictive of improvement after lung volume reduction surgery: use of CT morphometry. Chest. 2000;118:1240-1247.

30. Win $\mathrm{T}$, Lambrou $\mathrm{T}$, Hutton $\mathrm{BF}$, et al. ${ }^{18} \mathrm{~F}$-fluorodeoxyglucose positron emission tomography pulmonary imaging in idiopathic pulmonary fibrosis is reproducible: implications for future clinical trials. Eur J Nucl Med Mol Imaging. 2012;39: 521-528.

31. Ambrosini V, Zompatori M, De Luca F, et al. ${ }^{68} \mathrm{Ga}$-DOTANOC PET/CT allows somatostatin receptor imaging in idiopathic pulmonary fibrosis: preliminary results. J Nucl Med. 2010;51:1950-1955.

32. John AE, Luckett JC, Tatler AL, et al. Preclinical SPECT/CT imaging of $\alpha_{\mathrm{v}} \beta_{6}$ integrins for molecular stratification of idiopathic pulmonary fibrosis. $\mathrm{J} \mathrm{Nucl}$ Med. 2013;54:2146-2152.

33. Kessenbrock K, Plaks V, Werb Z. Matrix metalloproteinases: regulators of the tumor microenvironment. Cell. 2010;141:52-67.

34. Dunsmore SE, Saarialho-Kere UK, Roby JD, et al. Matrilysin expression and function in airway epithelium. J Clin Invest. 1998;102:1321-1331.

35. Razavian M, Nie L, Challa A, et al. Lipid lowering and imaging protease activation in atherosclerosis. $J$ Nucl Cardiol. 2014;21:319-328.

36. Bremer C, Bredow S, Mahmood U, Weissleder R, Tung CH. Optical imaging of matrix metalloproteinase-2 activity in tumors: feasibility study in a mouse model. Radiology. 2001;221:523-529.

37. Zhang X, Bresee J, Fields GB, Edwards WB. Near-infrared triple-helical peptide with quenched fluorophores for optical imaging of MMP-2 and MMP-9 proteolytic activity in vivo. Bioorg Med Chem Lett. 2014;24:3786-3790. 